Diversity of Research in Health Journal / Revue de la Diversité de la Recherche en Santé

Vol 4, January / Janvier 2021 - ISSN: 2561 -1666 DOI : 10.28984/drhj.v4i2.321

\title{
Opioid Crisis: A Qualitative Analysis of Financial Influences and Addiction
}

\section{N. Naccarato N. Wacker \& L. Gagnon}

\author{
DRHJ/RDRS 2021, 4, pp.1-16
}

\author{
Nicole Naccarato B.Sc.N. (c) \\ nnaccarato@laurentian.ca \\ School of Nursing, Faculty of Health \\ Laurentian University, Sudbury (ON), Canada \\ Noah Wacker B.Sc. \\ nwacker@laurentian.ca \\ Laurentian University, Sudbury (ON), Canada \\ Lissa Gagnon R.N, B.Sc.N, M.Sc.N \\ Assistant Professor, lgagnon@laurentian.ca \\ School of Nursing, Faculty of Health, \\ Laurentian University, Sudbury (ON), Canada
}

\begin{abstract}
Ontario is currently engaged in a public health crisis centered around the use of opioids. As the number of opioid-related harms continues to grow, reports have identified a need for further investigation and understanding of opioid use. The purpose of this qualitative study was to examine the differences in motives for opioid use amongst a diverse population. Theoretical thematic analysis and an interpretive framework were used to capture participants' lived experiences with opioid use. Data collection occurred by interviewing 14 participants at an Addictions Centre in Northern Ontario. Analysis of the results revealed a finding of financial influence in addiction and recovery. This insight is helpful in strengthening effective prevention and treatment strategies.
\end{abstract}

Keywords: opioids, cost, qualitative, motives for use, addiction, healthcare crisis

\section{Résumé}

La province de l'Ontario subit présentement une crise de santé publique centrée autour de l'usage des opioïdes. Depuis que le nombre de décès liés aux opioïdes augmente, les rapports exigent le besoin d'une enquête et compréhension plus approfondie aux personnes ayant un historique d'utilisation des opioïdes. Le but de cette recherche qualitative était d'examiner la différence entre les motifs pour l'usage de opioïdes parmi une population diversifiée à risque de dépendance et de décès liés aux opioïdes. La collecte de données a eu lieu par l'entremise d'entrevues de 14 participants à un centre de traitement de toxicomanie situé dans le nord de l'Ontario. Une analyse thématique théorique et un cadre interprétatif ont été utilisés pour analyser les expériences de participants qui ont vécu l'usage de opioïdes. L'analyse des résultats a identifié le lien entre les enjeux socioéconomiques en dépendance et de décès l'addiction et réhabilitation. Les résultats de cette recherche seront utiles pour améliorer des stratégies de prévention et d'intervention.

Mots clés : opioïdes, coût, qualitative, motifs pour l'usage, addiction, crise de santé publique

\section{Introduction}

The province of Ontario is currently engaged in a public health crisis centered around the use of prescription and illicit opioids. According to a report from Public Health Ontario (2018), the number of harms related to opioid misuse has risen steadily in Ontario for over a decade. The rate of apparent opioid related deaths increased rapidly from 6.2 to 10.6 per 100,000 Ontarians 
Diversity of Research in Health Journal / Revue de la Diversité de la Recherche en Santé Vol 4, January / Janvier 2021 - ISSN: 2561 -1666ＤOI : 10.28984/drhj.v4i2.321

from 2016 to 2019 (Public Health Ontario, 2020). As the number of opioid-related harms continue to grow, Canadian literature has identified a need for further investigation and understanding of opioid misuse (Belzak \& Halverson, 2018; Wallace et al., 2020).

Considerable evidence in substance abuse literature suggests that providing deeper understanding of the motives for opioid use is essential in developing effective prevention and treatment strategies (Cooper, Kuntsche, Levitt, Barber, \& Wolf, 2015; Hartwell, Back, McRaeClark, Shaftman, \& Brady, 2012). Interventions may be tailored to specifically target the motives underlying opioid use for a variety of individuals (Cooper et al., 2015). Among the substance abuse literature, the most reported motive for opioid use is pain (Barth, Moran-Santa Maria, Lawson, Shaftman, Brady, \& Black, 2013; Blevins, Lash, \& Abrantes, 2018; Kilwein, Hunt, \& Looby, 2018; McCabe, Veliz, Boyd, Schepis, McCabe, \& Schulenburg, 2019; McCabe \& Boyd, 2012; McCabe, Cranford, Boyd, \& Teter, 2007; Weiss et al., 2014). Other common motives for opioid use include to experiment (McCabe et al., 2007), get high (Barth et al., 2013; Kilwein et al., 2018; McCabe et al., 2007), avoid withdrawal (Blevins et al., 2018; Weiss et al., 2014), help with sleep (Barth et al., 2013; Blevins et al., 2018), be social (Blevins et al., 2018; Weiss et al., 2014), and to relieve anxiety, stress, or depression (Barth et al., 2013; Kilwein et al., 2018). Although motives for opioid use have been previously examined in substance abuse literature, there are scant studies examining motives through a qualitative lens. Utilizing a qualitative approach to examine motives for opioid use may uncover new themes that have not previously been addressed by the literature. In addition, a qualitative approach may provide further opportunity to examine the individual experience with opioid use and treatment.

Another gap in the literature is the sparse diversity of the population studied. The majority of substance abuse literature examines motives in adolescent or young adult populations such as high school or post-secondary students (Lin, Walton, Bonar, \& Blow, 2016; McCabe et al., 2019; McCabe et al., 2007; McCabe \& Boyd, 2012; Vosburg et al., 2016). According to reports from the Special Advisory Committee on the Epidemic of Opioid Overdoses (2018), the majority of opioid related harms affect individuals between the ages of 25-64. As a result of this demographic data, there is a need to examine motives for opioid use in populations inclusive of this age range. Additionally, the majority of literature examining motives for opioid use appears to only include those who use prescription opioids rather than illicit opioids such as heroin and street fentanyl (Barth et al., 2013; Jones, Spradlin, Robinson, \& Tragesser, 2014; McCabe et al., 2019; McCabe et al., 2007; McCabe \& Boyd, 2012; Weiss et al., 2014). According to Belzak and Halverson (2018) the Canadian opioid crisis is driven by both illicit and prescription opioids. Subsequently, there is a need to better understand individuals who use all forms of opioids. The purpose of this research study is to qualitatively examine individuals' experiences with opioids, specifically focusing on their motives for use. Collecting data in Northern Ontario regarding the opioid crisis will also add to the diversity of knowledge surrounding opioid addiction and motives for use. 
Diversity of Research in Health Journal / Revue de la Diversité de la Recherche en Santé

Vol 4, January / Janvier 2021 - ISSN: 2561 -1666 DOI : 10.28984/drhj.v4i2.321

\section{Methods}

\section{Ethics}

Ethical approval to conduct this study was sought and granted through the Research and Ethics Board of Laurentian University.

\section{Setting and Sample}

This research study was conducted at an Addictions Treatment Centre in Northern Ontario. One of the main priorities of this centre was the treatment of opioid addiction through methadone and suboxone programs. At the time of the study there were approximately 50 patients receiving treatment daily. The study relied on purposive sampling. Eligibility criteria included having personal experience with the use of prescription or illicit opioids, the ability to speak and understand English, and the ability to provide informed consent. The study did not exclude participants on the attribute of age, ethnicity, or culture. Participants were given the option to interview before or after receiving their scheduled dose of methadone or suboxone. Previous studies indicate that participants' treatment of methadone or suboxone should not affect their cognitive ability to accurately respond to interview questions (Anderson \& McNair, 2018).

\section{Data Collection}

Recruitment for the study occurred over a span of three weeks. Paper copies of the invitation to participate were posted on a message board at the centre and left at the front desk for potential participants to take home. This process provided individuals with time to consider if they were eligible and willing to participate in the study.

The researchers interviewed participants who responded to the invitation to participate and met the eligibility criteria. Data was collected utilizing semi-structured interviews over a total of four days. Members of the research team met with participants in a private setting in the centre. The interview process began by reaffirming consent with the participant followed by the collection of demographic data which included age, gender, culture, and/or ethnicity. All interviews were audio recorded. The research team created and used an interview guide with open-ended questions derived from relevant literature to determine participant beliefs and personal motives for opioid use. For example, one question inquired about the participant's initial use with opioids. All participants were interviewed once. The interview durations ranged from 15 to 30 minutes, however, they were not limited to a specific time period. At no time did a participant indicate the need to withdraw from an interview. The research team continued to interview participants until data saturation was reached. The interviews were then transcribed verbatim by the research team.

Participant confidentiality was maintained at all times throughout the study. Participants were able to withdraw from the study or decline to respond to questions without consequence to their treatment.

\section{Interview Safety Precautions}

During the interview process, specific steps were taken to ensure the safety of participants. The first step required language sensitivity. Following the suggestions of Clark 
(2017), the research team avoided the use of language that could label the participants in order to support positive well-being.

The second step to maintain participant safety was centred around modifying the interview process. Given the sensitive nature of the interview questions, there was a need to address potential risk for emotional distress and relapse. Based on the literature, the research team developed plans to manage emotional distress and triggers (Dempsey, Dowling, Larkin, \& Murphy, 2016). The decision was made to conduct interviews at the centre on days when it was fully staffed to ensure the safety of all participants. The centre had healthcare professionals on site who were aware of the study and were prepared to provide immediate assistance if required as they had specialized training in addiction and supportive treatment. A question addressing participants' current mental well-being and desire to use opioids was included at the end of the interview. If a participant felt triggered to use opioids, they were encouraged to discuss it immediately with the healthcare professionals at the centre. Participants were also provided with a list of external resources which they could access at any time. The resources addressed addiction, trauma, and mental health.

The research team also developed a distress protocol which detailed instructions in the event that a participant experienced any form of distress. The protocol was adapted from literature on managing distress in qualitative interviewing (Dempsey et al., 2016; Drauker, Martsolf, \& Poole, 2009; Haigh \& Witham, 2015). The protocol addressed elements such as grounds for interview termination, reasons to initiate the protocol, and methods to manage immediate and continued responses.

An additional initial step taken by the research team was to develop their own knowledge of the sensitive interview process. Dempsey et al. (2016) provide guidelines for sensitive interviewing in qualitative research which include methods such as empathetic listening, developing trusting rapport with participants, having thorough knowledge of interview questions to allow for free-flowing conversation, and maintaining anonymity and confidentiality.

Another issue that was addressed by the research team was duty to report. Duty to report refers to the ethical responsibility of immediately articulate information regarding harm to oneself or others to the respective authority (Paquette \& Ross, 2014). All information was kept confidential with the exception of statements that required a duty to report. A statement was included in the consent form which addressed the information requiring a duty to report.

\section{Data Analysis}

This paper presents a component of a larger research project examining motives for the use of opioids. To analyze the data, the research team utilized Braun and Clarke's (2006) inductive thematic analysis to serve as a guide for data collection and categorization of themes within the participants' narratives. In addition, Polio, Henley, and Thompson's (2006) approach to existential phenomenology was utilized to as the interpretive framework. The research team opted to use this dual approach, inductive thematic analysis and existential phenomenology, as a means to enhance the qualitative rigor of the study. 
Following Polio et al.'s (2006) approach, it was essential for the research team to ensure that interpretation of the data remained close to the participants' narratives. To ensure these criteria were met, the research team utilized two techniques suggested by Polio et al. (2006) prior to data analysis. The first involved a bracketing technique in which members of the research team came together and openly reflected upon their potential biases, perspectives, and clarified any preconceptions regarding opioid use. The background of the research team consisted of nursing and sciences in which individual perspectives varied amongst members. The second technique involved the research team conducting an interpretive research group in which transcripts were selected at random, read aloud, and then discussed amongst the members of the group. By following these techniques, the research team ensured the focus remained on participants' narratives thus increasing the qualitative rigor of the study.

The research team then utilized Braun and Clarke's (2006) inductive thematic analysis to identify themes and patterns within the data. As inductive thematic analysis is driven by the data (Braun \& Clarke, 2006), participants' narratives were coded without trying to fit them into existing frames. Therefore, the research team individually read the transcripts line-by-line and grouped excerpts into equivalent or related trends. Once this step was completed the research team met to collate pertinent excerpts from the participants' narratives. A final third reading of the data occurred to ensure the data formed coherent themes and patterns. Where there was disagreement regarding whether a data excerpt aligned with a specific theme, it was discussed amongst the research team until consensus was reached.

\section{Findings}

\section{Description of Participants}

Participants were patients of the centre seeking either methadone or suboxone treatment. The final sample included a total of 14 participants. Nine participants identified as female and five identified as male. Participant ages ranged from 26-58 years old. Participants self-identified as Indigenous, Caucasian, French Canadian, and Italian.

\section{Presentation of Results}

Thematic analysis segmentation resulted in 19 excerpts which yielded a theme of financial influence throughout the lived experience of opioid addiction and recovery of participants. The excerpts were analyzed further and grouped into patterns representing the different roles financial influence played throughout the participants' experience with opioids as presented in Figure 1. This theme was sub-divided into four patterns: Funds As a Motive to Seek Methadone or Suboxone Treatment, Funds As a Motive to Continue to Use Opioids, Funds As a Motive to Maintain Compliance with Treatment, and Funds As a Motive to Seek Alternate Substances. 


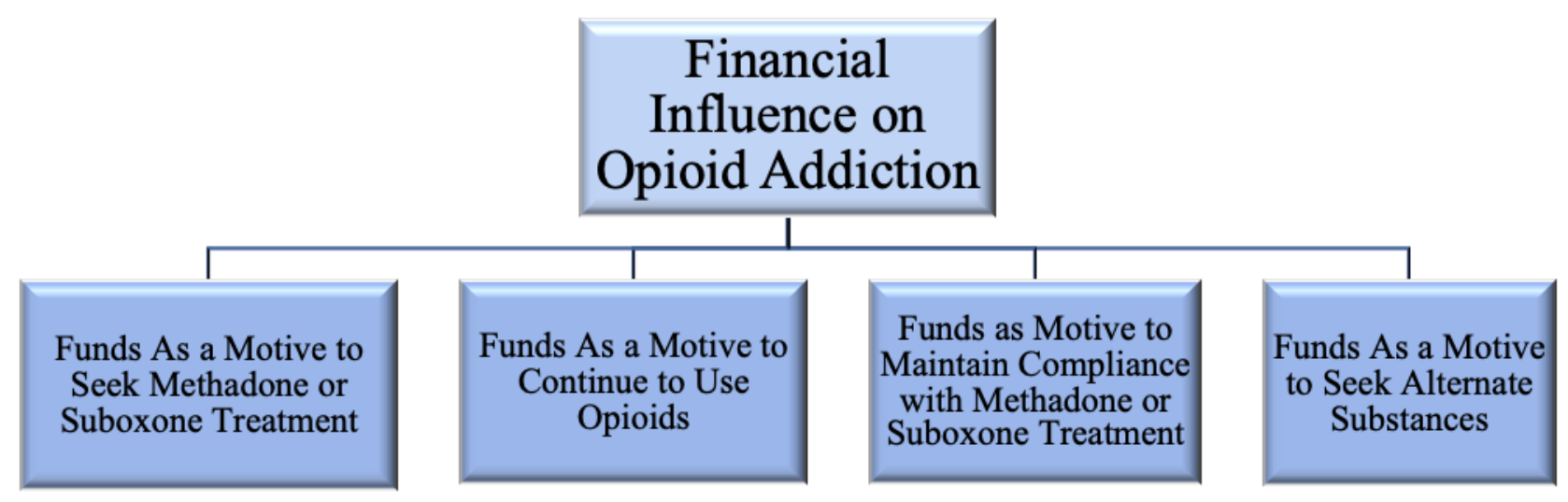

Figure 1. Theme and patterns of financial influence on opioid addiction.

Pattern 1. The first pattern was Funds As a Motive to Seek Methadone or Suboxone Treatment. During interviews the participants easily provided descriptions about the financial burden of maintaining their opioid addiction and the associated stress. Participants shared that they no longer had the resources to purchase opioids and therefore were motivated to seek treatment as a last resort.

I003: "Once you start running low on money or food you know, it's like you can't buy it [opioids], you physically can't."

Some participants stated that their reason for seeking treatment was the realization of how high the cost of maintaining their opioid addiction was becoming. These participants continued to describe the stress related to this reality.

I014: If it wasn't so expensive [...] I probably would've kept on [abusing opioids]."

I004: "Just my life was like becoming unmanageable [...] Financially and mentally."

Other participants expressed the view that their motivation to seek treatment stemmed from the emotional burden of being financially dependent on either family members or friends to maintain their addiction. Some participants also expressed guilt as they believed they financially burdened their family and or friends.

I001 [Mom] was living with me and every time I was sick and needed money for a pill all my money was gone so I would turn to her. She would offer to help but I shouldn't have took it because I would make her broke. And everyone around me, family too. 
Diversity of Research in Health Journal / Revue de la Diversité de la Recherche en Santé Vol 4, January / Janvier 2021 - ISSN: 2561 -1666 DOI : 10.28984/drhj.v4i2.321

In contrast, a few participants revealed that their motivator to seek treatment was the mental burden of financially supporting their own opioid addiction in addition to other dependants.

I012: "I actually had a complete mental breakdown, I was actually working and supporting three other people... I couldn't take it anymore. I just had a complete mental breakdown."

Pattern 2. The second pattern was Funds as a Motive to Continue to Use Opioids. Participants discussed how having the financial resources to fund their addiction was a motivator to seek and use opioids. For some participants the availability of money provoked them to use opioids.

I003: "So like if they do have money, they, they will want to go out and use like it is so hard to explain."

I008: "Right now I can't have money. I can't hold on to money... It's one trigger lately I've been having hard time with money... just 20 bucks and I want to go run."

Some participants also suggested that if money was replaced with resources such as food, clothes, and stable housing the motive to seek or use opioids would be removed.

I003: If people are committed and they have the mindset to stay off, it will help them. It will. But if they get you know, money in their hands, they're going to go out and use. Like let's say they win the lottery

for five grand, it's going to drugs. Whether they are on this program or not. So like you have to almost take away everyone's money, put them on the system, and they will do good.

Some participants were aware of their spending habits, while others did not realize how much they were spending until after seeking methadone or suboxone treatment.

I009: “I didn't realize then like how much money was getting spent on drugs and... Yeah they were a lot."

I003: "They know that they can waste $\$ 2000$ on it [opioids] ... I was spending $\$ 120$ a day on fentanyl patches."

Pattern 3. The third pattern was Funds as Motive to Maintain Compliance with Methadone or Suboxone Treatment. The low cost of methadone and suboxone treatment acted as a motive for some participants to continue receiving treatment. Several participants stated that after they began with methadone or suboxone, the financial toll of their addiction was greatly reduced. 
Participants were pleased that they no longer had to continue with behaviours in order to fund their addiction.

I014: “I think it's lifesaving for a lot of people... you don't suffer the negative things in life of ... no money and what you have to do for money in order to get the stuff which is ridiculously expensive..."

In addition, no longer having to fund an addiction allowed participants to begin to stabilize other aspects of their lives. Participants also found the psychological strain of knowing they must take part in undesirable activities to get money to buy opioids was also alleviated after receiving treatment.

I013: I don't have to go steal or whatever fetching it and I come and get it and that's it, it keeps me normal... I've been in and out of prison in my past, I mean I've spent half of my life in and out of jail. And finally, I straightened my life out.

Pattern 4. The final pattern was Funds As a Motive to Seek Alternate Substances.

Several participants explained how the cost of different substances acted as a motive for them to seek cost-efficient substances. Participants noted that the long lasting effect of opioids compared to the short lived effects of other similarly priced substances such as stimulants was a reason they continued to use opioids. In this sense, many participants viewed opioids as cost effective which was a reason they gravitated towards these types of substances.

I011: “Cocaine is $\$ 100$ a gram too. It's not cheap. I mean I can do three to four hundred worth of cocaine injection in a night $[\ldots]$ whereas no one can do that much heroin in a night."

Additionally, some participants found that the varying costs of different opioids was a motive to try new opioids, especially if they were less expensive. The cost of opioids also acted as a motive to try using different routes of intake in order to achieve greater effects for the same cost.

I009: "[Oxycodone pills] were expensive. But then, instead of just sniffing it we started smoking them."

I012: $\quad$ For me it was mostly what was cheaper... I could always get oxys but why would I buy one 80mg pill when I can get two [dilaudid or hydromorph] pills that would have the same effect and the same duration you know for the same price. 
Diversity of Research in Health Journal / Revue de la Diversité de la Recherche en Santé Vol 4, January / Janvier 2021 - ISSN: 2561 -1666ＤOI : 10.28984/drhj.v4i2.321

\section{Discussion}

Based on participant narratives, it was clear that financial influence was apparent throughout their lived experiences involving opioid addiction and recovery. This theme was unexpected by the research team. Despite none of the interview prompts alluding to finances, this topic was commonly brought up by participants. Although financial influence is mentioned in previous substance abuse and opioid literature, it is seldom presented as a motive for the use of opioids. The unexpectedness of this finding may be related to the qualitative nature of the research work. Qualitative methods provide the context for researchers to examine participant experiences as a whole rather than a specific aspect of the experience (Ivey, 2012). Through a qualitative methodology the researchers determined that finances influence participants' experiences with opioids. Based on this study's findings the authors suggest that financial factors may influence multiple aspects of the experience such as seeking and using opioids, choosing types of opioids and routes of administration, making decisions to seek treatment, and remaining compliant with treatment.

The findings also demonstrate how finance can influence individuals who use opioids to seek methadone or suboxone treatment. This finding supports previous literature which highlights how the cost of opioids can act as a motivating factor to initially seek treatment (Amini-Rarani, Khedmati Morasae, Pashaei, \& Moeeni, 2020; Bansal, Sidana, \& Mehta, 2019; Andrews, Kramer, Klumper, \& Barrington, 2012; Parmar, Patil, Sarkar, \& Rao, 2018). In addition to demonstrating how a lack of funds can motivate opioid users to seek treatment initially, the findings also suggest that finance influences opioid users to continue with treatment. This suggests that the role finance plays in addiction should be examined throughout the entirety of the recovery process. Methadone and suboxone treatment are more affordable than illicit opioids in Ontario (Zaric, Brennan, Varenbut \& Daiter, 2012). The authors of this study believe it is of interest to explore how the role of finance changes in locations where methadone and suboxone treatment is more expensive.

The pattern of findings demonstrating Funds as a Motive to Continue to Use Opioids describes how some participants viewed the availability of money as a motivator or trigger to seek and use opioids. This finding is supportive of previous substance abuse literature on the association between finance and drug use. One phenomenon suggested by previous literature is termed the "check effect" which was described as an increase in illicit substance use and related harms at times in which individuals receive income assistance (Hoj, Jacka, Minoyan, Bussiere, \& Bruneau, 2020; Otterstatter, Amlani, Guan, Richardson, Buxton, 2016; Rosen, 2011). While some authors (Rosen, 2011) suggest that income assistance has little impact on overall drug use, other authors contend that any sudden financial gain may be a cue for individuals to use drugs (Hamilton \& Potenza, 2012; Obrien, Childress, McLellan, \& Ehrman, 1990). The findings from the current study support financial gain as a cue to use opioids as participants stated that receiving any form of money was a motivator to seek and use opioids. As a result, the authors suggest that the availability of money plays a vital role in opioid use and methadone and suboxone treatment. Furthermore, individuals should have continued access to funding supports 
as well as increased ongoing assistance from healthcare services. Another finding was that some participants in this study were aware of their association of financial gain and opioid use. Increased awareness of this personal association may provide individuals in methadone or suboxone treatment with the opportunity to self-identify and report to friends, family, or healthcare professionals for assistance when they are at an increased risk for relapse.

Another relevant process is "delay discounting" which is described in literature as the process where an individual places high value on immediate short-term rewards and "discounts" the value of long-term rewards (Daugherty \& Brase, 2010; Hamilton \& Potenza, 2012). Previous studies have suggested that those who abuse substances are more likely to delay discount than non-abusing controls (Black \& Rosen, 2011; Daugherty \& Brase, 2010; Hamilton \& Potenza, 2012). This phenomenon has also held true in studies specific to opioid use (Giordano, Bickel, Loewenstein, Jacobs, Marsch, Badger, 2002; Robles, Huang, Simpson, \& McMillan, 2011). Many studies have suggested a positive association between delay discounting and financial mismanagement in substance abusers (Black \& Rosen, 2011; Hamilton \& Potenza, 2012). The findings of the current study support the existing literature as some of the participants did not recognize the long-term consequences of their spending until their financial situation became unmanageable.

Given the findings supporting delay discounting and the overall theme of financial influence, the researchers question how individuals who use opioids would be affected by the social determinants of health. It is understandable that financial influence is directly related to income and socioeconomic status. Previous literature has suggested that wealthier individuals may be less likely to delay discount (Hamilton \& Potenza, 2012). Therefore, it may be possible that individuals who use opioids and are of low income are more likely to associate the availability of money as a cue for drug use. Social and economic factors are also known to exacerbate risk behavior and affect the overall health of drug users (Galea \& Vlahov, 2002). According to Van Draanen, Tsang, Mitra, Karamouzian and Richardson (2020), socioeconomic marginalization is associated with an increased likelihood of overdose. Socioeconomic factors such as income and housing have also been suggested to amplify the previously mentioned check effect phenomenon, leading to increased negative health and financial outcomes (Goedel, Green, Viner-Brown, Rich, \& Marshall, 2019). As displayed by the findings of this study, opioid addiction can heavily affect an individual's socioeconomic status. Therefore, the risk of delay discounting and opioid related harm may change over an individual's life as their socioeconomic status changes. The researchers suggest a need for further research on the connections between the social determinants of health, financial influence, and opioid-related use and harms. The researchers also recommend a need for further investigation regarding the efficacy of programs focused on improving financial management specific to opioid misuse. Finally, the authors of this study emphasize the need of effective interventions that serve to reduce potential health inequities associated with financial influence.

Recent Canadian reports have detailed a dramatic increase in opioid related harms since government mandated quarantines began in March of 2020 as a result of COVID-19 (Canadian 
Centre on Substance Use and Addiction, 2020; Public Health Agency of Canada, 2020). Additionally, the Canadian economy has been greatly affected with current employment down approximately 1.3 million as result of the pandemic (Statistics Canada, 2020). The government has created initiatives to financially support Canadian citizens such as the Canadian Emergency Response Benefit (Government of Canada, 2020). Given the findings of this study and supporting evidence in the literature such as delay discounting and the social determinants of health, it is unknown how the availability of money and socioeconomic status will affect individuals who use opioids. A recent report from the Canadian Centre on Substance Use and Addiction (2020) found that individuals who use substances such as opioids have reported a loss of social connection and support as well as an increase in isolation and anxiety during the COVID-19 pandemic (Canadian Centre on Substance Use and Addiction, 2020). Based on the current evidence and the findings of this study, the authors contend there is an urgent need to discover interventions to effectively support individuals who use opioids during the COVID-19 pandemic.

\section{Limitations}

Participants' responses may have been affected by a passage of time as they spoke of past experiences. The sample population in this study included individuals from one treatment centre and thus reflects the lived experience of one sample. However, the findings may not be generalizable to other treatment Centres.

\section{Conclusions}

Using interviews to collect data was found to be an effective method of obtaining information on participants' experiences with opioids. All participants shared that they were comfortable with the interview process. Several participants also explained that they found the process to be therapeutic and rewarding and stated they were thankful for the opportunity to articulate their story. Using a qualitative approach with precautions and supports can create a safe environment to collect data. Similar qualitative approaches should continue to be used synergistically with quantitative data to understand the motives for opioid use and treatment.

This qualitative research study interviewed individuals seeking methadone and suboxone treatment at an Addictions Treatment Centre in Northern Ontario. Analysis of participants' experiences with opioids revealed financial influence as a recurring theme throughout their addiction and recovery. The theme of financial influence was subdivided into four patterns: Funds As a Motive to Seek Methadone or Suboxone Treatment, Funds As a Motive to Continue to Use Opioids, Funds As a Motive to Maintain Compliance with Treatment, and Funds As a Motive to Seek Alternate Substances. These findings will contribute to a larger body of empirical knowledge that can be beneficial in preventing and treating opioid addiction. There is a progressive need for future studies to gain a better understanding of individuals and opioid crises. 
Diversity of Research in Health Journal / Revue de la Diversité de la Recherche en Santé Vol 4, January / Janvier 2021 - ISSN: 2561 -1666 DOI : 10.28984/drhj.v4i2.321

\section{Acknowledgments}

The authors thank the members at the Addictions Treatment Centre for their support of the study. The authors also thank participants for volunteering and sharing their experiences. The authors would also like to thank Sophie Hébert for her contributions and support of the project. In addition, the research team recognizes that the study occurred on the Robinson-Huron Treaty of 1850 territory and acknowledges the traditional lands of the Atikameksheng Anishnawbek and Wahnapitae First Nations.

\section{References}

Amini-Rarani, M., Khedmati Morasae, E., Pashaei, T., \& Moeeni, M. (2020). Redemption from plight: A qualitative study on reasons behind treatment decisions among Iranian male opioid users. Substance Abuse Treatment, Prevention, and Policy, 15(1). doi:10.1186/s13011-020-00299-x

Anderson, E., \& McNair, L. (2018). Ethical issues in research involving participants with opioid use disorder. Therapeutic Innovation \& Regulatory Science, 52(3), 280-284. doi:10.1177/2168479018771682

Andrews, D., Kramer, R., Klumper, L., \& Barrington, C. (2012). A qualitative exploration of individuals' motivators for seeking substance user treatment. Substance Use \& Misuse, 47(11), 1224-1233. doi:10.3109/10826084.2012.700473

Bansal, S., Sidana, A., \& Mehta, S. (2019). Pathways to care and reasons for treatment-seeking behavior in patients with opioid dependence syndrome: An exploratory study. Journal of Mental Health and Human Behaviour, 24(1), 8. doi:10.4103/jmhhb.jmhhb_40_18

Barth, K. S., Maria, M. M., Lawson, K., Shaftman, S., Brady, K. T., \& Back, S. E. (2013). Pain and motives for use among non-treatment seeking individuals with prescription opioid dependence. The American Journal on Addictions, 22(5), 486-491. doi:10.1111/j.1521-0391.2013.12038.x

Belzak, L., \& Halverson, J. (2018). The opioid crisis in Canada: a national perspective. Health Promotion and Chronic Disease Prevention in Canada, 38(6), 224-233. https://doi.org/10.24095/hpcdp.38.6.02

Black, A. C., \& Rosen, M. I. (2011). A money management-based substance use treatment increases valuation of future rewards. Addictive Behaviors, 36(1-2), 125-128. doi:10.1016/j.addbeh.2010.08.014

Blevins, C. E., Lash, S. J., \& Abrantes, A. M. (2018). Adapting substance use motives measures for a clinical population of opiate, alcohol, and stimulant users. Addiction Research \& Theory, 26(2), 151-158. doi:10.1080/16066359.2017.1338689

Braun, V., Clarke, V. (2006). Using thematic analysis in psychology. Qualitative Analysis in Psychology, 3(2), 77-101. doi: 10.1191/1478088706qp063oa

Canadian Centre on Substance Use and Addiction. (2020). Impact of the COVID-19 Pandemic 
Diversity of Research in Health Journal / Revue de la Diversité de la Recherche en Santé Vol 4, January / Janvier 2021 - ISSN: 2561 -1666 DOI : 10.28984/drhj.v4i2.321

on People Who Use Substances: What We Heard. Ottawa: Canadian Centre on Substance Use Addiction. Retrieved from https://www.ccsa.ca/sites/default/files/2020-07/CCSA-COVID-19-Impacts-on-PeopleWho-Use-Substances-Report-2020-en.pdf

Clark, L. (2017). Interviewing Vulnerable Populations. The International Encyclopedia of Communication Research Methods, 1-2. doi:10.1002/9781118901731.iecrm0126

Cooper, M. L., Kuntsche, E., Levitt, A., Barber, L. L., \& Wolf, S. (2015). Motivational models of substance use. Oxford Handbooks Online. doi:10.1093/oxfordhb/9780199381678.013.017

Daugherty, J. R., \& Brase, G. L. (2010). Taking time to be healthy: Predicting health behaviors with delay discounting and time perspective. Personality and Individual Differences, 48(2), 202-207. doi:10.1016/j.paid.2009.10.007

Dempsey, L., Dowling, M., Larkin, P., \& Murphy, K. (2016). Sensitive interviewing in qualitative research. Research in Nursing \& Health, 39(6), 480-490. doi:10.1002/nur.21743

Draucker, C. B., Martsolf, D. S., \& Poole, C. (2009). Developing distress protocols for research on sensitive topics. Archives of Psychiatric Nursing, 23(5), 343-350. doi:10.1016/j.apnu.2008.10.008

Galea, S., \& Vlahov, D. (2002). Social determinants and the health of drug users: socioeconomic status, homelessness, and incarceration. Public Health Reports, 117(1), S135-S145. Retrieved from https://www.ncbi.nlm.nih.gov/pmc/articles/PMC1913691/

Giordano, L., Bickel, W., Loewenstein, G., Jacobs, E., Marsch, L., \& Badger, G. (2002). Mild opioid deprivation increases the degree that opioid-dependent outpatients discount delayed heroin and money. Psychopharmacology, 163(2), 174-182. doi:10.1007/s00213002-1159-2

Goedel, W.C., Green, T.C., Viner-Brown, S., Rich, J.D., Marshall, B.D.L. (2019). Increased overdose mortality during the first week of the month: Revisiting the "check effect" through a spatial lens. Drug and Alcohol Dependence, 197, 49-55. https://doi.org/10.1016/j.drugalcdep.2018.12.024

Government of Canada. (2020). Canada Emergency Response Benefit (CERB). Ottawa: Government of Canada. Retrieved from https://www.canada.ca/en/services/benefits/ei/cerb-application.html

Haigh C. \& Witham G. (2015). Distress protocol for qualitative data collection. Retrieved from https://www.mmu.ac.uk/media/mmuacuk/content/documents/rke/Advisory-DistressProtocol.pdf

Hamilton, K. R., \& Potenza, M. N. (2012). Relations among delay discounting, addictions, and money mismanagement: Implications and future directions. The American Journal of Drug and Alcohol Abuse, 38(1), 30-42. doi:10.3109/00952990.2011.643978

Hartwell, K. J., Back, S. E., McRae-Clark, A. L., Shaftman, S. R., \& Brady, K. T. (2012). 
Diversity of Research in Health Journal / Revue de la Diversité de la Recherche en Santé Vol 4, January / Janvier 2021 - ISSN: 2561 -1666ＤOI : 10.28984/drhj.v4i2.321

Motives for using: A comparison of prescription opioid, marijuana and cocaine dependent individuals. Addictive Behaviors, 37(4), 373-378. doi:10.1016/j.addbeh.2011.11.014

Høj, S. B., Jacka, B., Minoyan, N., Bussière, P., \& Bruneau, J. (2020). Deconstructing the 'cheque effect': Short-term changes in injection drug use after receiving income assistance and associated factors. Addiction, 1-12. doi:10.1111/add.15192

Ivey, J. (2012). The value of qualitative research methods. Pediatric Nursing, 38(6), 319. Retrieved from https://link-galecom.librweb.laurentian.ca/apps/doc/A314442879/AONE?u=subd78095\&sid=AONE\&xi $\mathrm{d}=\mathrm{fb} 0 \mathrm{ee} 7 \mathrm{fc}$

Jones, R.E., Spradlin, A., Robinson, R.J., \& Tragesser, S.L. (2014). Development and Validation of the Opioid Prescription Medication Motives Questionnaire: A Four-Factor Model of Reasons for Use. Psychology of Addictive Behaviors, 28(4), 1290-1296. https://doi.org/10.1037/a0037783

Lin, L. A., Walton, M. A., Bonar, E. E., \& Blow, F. C. (2016). Trajectories of nonmedical use of prescription opioids among adolescents in primary care. Addiction Research \& Theory, 24(6), 514-520. doi:10.1080/16066359.2016.1178244

Kilwein, T.M., Hunt, P., Looby, A. (2018). A Descriptive Examination of Nonmedical Fentanyl Use in the United States: Characteristics of Use, Motives, and Consequences. Journal of Drug Issues, 48(4), 409-420. https://doi.org/10.1177/0022042618765726

McCabe, S. E., \& Boyd, C. J. (2012). Do motives matter? Nonmedical use of prescription medications among adolescents. PsycEXTRA Dataset. doi:10.1037/e542612012-004

McCabe, S. E., Cranford, J. A., Boyd, C. J., \& Teter, C. J. (2007). Motive, source, and route of administration for Nonmedical use of prescription opioids measure. PsycTESTS Dataset. doi:10.1037/t17822-000

McCabe, S. E., Veliz, P. T., Boyd, C. J., Schepis, T. S., McCabe, V. V., \& Schulenberg, J. E. (2019). A prospective study of nonmedical use of prescription opioids during adolescence and subsequent substance use disorder symptoms in early midlife. Drug and Alcohol Dependence, 194, 377-385. doi:10.1016/j.drugalcdep.2018.10.027

O'Brien, C. P., Childress, A. R., McLellan, T., \& Ehrman, R. (1990). Integrating systematic cue exposure with standard treatment in recovering drug dependent patients. Addictive Behaviors, 15(4), 355-365. doi:10.1016/0306-4603(90)90045-y

Ontario Agency of Health Protection and Promotion (Public Health Ontario). (2020). Interactive opioid tool: opioid-related morbidity and mortality in Ontario. Toronto, ON: Queen's Printer for Ontario. Retrieved from https://www.publichealthontario.ca//media/documents/ncov/main/2020/08/substance-use-related-harms-disruption.pdf?la=en

Ontario Agency for Health Protection and Promotion (Public Health Ontario); Office of the Chief Coroner; Ontario Forensic Pathology Service; Ontario Drug Policy Research Network. (2018). Opioid mortality surveillance report: analysis of opioid-related deaths in Ontario July 2017-June 2018. Toronto, ON: Queen's Printer for Ontario. Retrieved 
Diversity of Research in Health Journal / Revue de la Diversité de la Recherche en Santé Vol 4, January / Janvier 2021 - ISSN: 2561 -1666ＤOI : 10.28984/drhj.v4i2.321

from__ https://www.publichealthontario.ca/-/media/documents/O/2019/opioid-mortalitysurveillance-report.pdf?la=en

Otterstatter, M. C., Amlani, A., Guan, T. H., Richardson, L., \& Buxton, J. A. (2016). Illicit drug overdose deaths resulting from income assistance payments: Analysis of the 'check effect' using daily mortality data. International Journal of Drug Policy, 33, 83-87. doi:10.1016/j.drugpo.2016.05.010

Paquette, E.T., \& Ross, L.F. (2014). The Moral and Legal Need to Disclose Despite a Certificate of Confidentiality. American Journal of Bioethics, 14(10), 51-53. doi:10.1080/15265161.2014.947817

Parmar, A., Patil, V., Sarkar, S., \& Rao, R. (2017). An observational study of treatment seeking users of natural opiates from India. Substance Use \& Misuse, 53(7), 1139-1145. doi:10.1080/10826084.2017.1400564

Pollio, H., Henley, T., Thompson, C.B. (2006). The Phenomenology of Everyday Life. Cambridge University Press, Cambridge, New York.

Public Health Agency of Canada. (2020, May 29). Statement from the chief public health officer of Canada on COVID-19. Retrieved from_https://www.canada.ca/en/publichealth/news/2020/05/statement-from-the-chief-public-health-officer-of-canada-on-covid198.html

Robles, E., Huang, B. E., Simpson, P. M., \& McMillan, D. E. (2011). Delay discounting, impulsiveness, and addiction severity in opioid-dependent patients. Journal of Substance Abuse Treatment, 41(4), 354-362. doi:10.1016/j.jsat.2011.05.003

Rosen, M.I. (2011). The 'check effect' reconsidered. Addiction, 106, 1071-1077. doi:10.1111/j.1360-0443.2011.03409.x

Special Advisory Committee on the Epidemic of Opioid Overdoses. (2018). Interactive Opioid Tool: Opioid-related morbidity and mortality in Ontario. Web-based Report. Ottawa, ON: Public Health Agency of Canada. Retrieved from https://www.publichealthontario.ca/en/data-and-analysis/substance-use/interactiveopioid-tool\#/ageSex

Statistics Canada. (2020). Labour Force Survey, July 2020. Ottawa: Government of Canada. Retrieved from https://www150.statcan.gc.ca/n1/daily-quotidien/200807/dq200807aeng.htm

Van Draanen, J., Tsang, C., Mitra, S., Karamouzian, M., \& Richardson, L. (2020).

Socioeconomic marginalization and opioid-related overdose: A systematic review. Drug and Alcohol Dependence, 214, 108127. https://doi.org/10.1016/j.drugalcdep.2020.108127

Vosburg, S. K., Eaton, T. A., Sokolowska, M., Osgood, E. D., Ashworth, J. B., Trudeau, J. J., ...Katz, N. P. (2015). Prescription opioid abuse, prescription opioid addiction, and heroin abuse among adolescents in a recovery high school: A pilot study. Journal of Child \& Adolescent Substance Abuse, 25(2), 105-112. doi:10.1080/1067828x.2014.918005

Wallace, B., Van Roode, T., Pagan, F., Phillips, P., Wagner, H., Calder, S., ... Hore, D. (2020). 
Diversity of Research in Health Journal / Revue de la Diversité de la Recherche en Santé Vol 4, January / Janvier 2021 - ISSN: 2561 -1666 DOI : 10.28984/drhj.v4i2.321

What is needed for implementing drug checking services in the context of the overdose crisis? A qualitative study to explore perspectives of potential service users. Harm Reduction Journal, 17(1). doi:10.1186/s12954-020-00373-4

Weiss, R. D., Potter, J. S., Griffin, M. L., McHugh, R. K., Haller, D., Jacobs, P., ... Rosen, K. D. (2014). Reasons for opioid use among patients with dependence on prescription opioids: The role of chronic pain. Journal of Substance Abuse Treatment, 47(2), 140145. doi:10.1016/j.jsat.2014.03.004

Zaric, G. S., Brennan, A. W., Varenbut, M., \& Daiter, J. M. (2012). The cost of providing methadone maintenance treatment in Ontario, Canada. The American Journal of Drug and Alcohol Abuse, 38(6), 559-566. https://doi.org/10.3109/00952990.2012.694518 